\title{
Detection of active LTR-retrotransposon via eccDNA analysis in Helianthus annuus L., Arabidopsis thaliana and triticale
}

\author{
Merkulov P. ${ }^{1 *}$, Omarov M. ${ }^{1,2}$, Gvaramiya S. ${ }^{1}$, Kirov I. ${ }^{1}$ \\ ${ }^{1}$ All-Russia Research Institute of Agricultural Biotechnology, Moscow, Russia \\ ${ }^{2}$ National Research University Higher School of Economics, Moscow, Russia \\ * email: paulmerkulov97@gmail.com
}

LTR-retrotransposons present a subclass of mobile genetic elements and can produce extrachromosomal circular DNA (eccDNA) as a byproduct of their life-cycle. Recent studies showed that the amount of LTR-retrotransposon eccDNA molecules is individual for each element and often correlates with the transposition ability. In this way, eccDNA can be used for experimental detection of active retrotransposons in plants as an alternative to whole-genome sequencing. In our study, we aimed to reveal active retrotransposons in Arabidopsis thaliana, Helianthus annuus L. and triticale ( $\times$ Triticosecale Wittmack). Later two species have relatively large and repeat-rich genomes. Total genomic DNA was isolated and treated with plasmid-safe exonuclease for eccDNA enrichment. Then we performed a Rolling Circle Amplification (RCA) reaction followed by inverse PCR with specific primers. In $H$. annuus we detected eccDNA for LTR-retrotransposon (named Gagarin) located on chromosome 1. For triticale, we detected a retrotransposon (named $M I G$ ) located on chromosome 7B and produced eccDNA molecules. Using RT-PCR analysis and direct RNA Nanopore sequencing we also detected different isoforms for these retrotransposons. In conclusion, eccDNA detection is an accurate method for the identification of active LTRretrotransposons in large and repetitive plant genomes.

Acknowledgements: The reported study was funded by RFBR, project number 20-3470032. 